\title{
Control of obstacle climbing in the cockroach, Blaberus discoidalis. I. Kinematics
}

Accepted: 18 December 2001 / Published online: 31 January 2002 (C) Springer-Verlag 2002

\begin{abstract}
An advantage of legged locomotion is the ability to climb over obstacles. We studied deathhead cockroaches as they climbed over plastic blocks in order to characterize the leg movements associated with climbing. Movements were recorded as animals surmounted $5.5-\mathrm{mm}$ or $11-\mathrm{mm}$ obstacles. The smaller obstacles were scaled with little change in running movements. The higher obstacles required altered gaits, leg positions and body posture. The most frequent sequence used was to first tilt the front of the body upward in a rearing stage, and then elevate the center of mass to the level of the top of the block. A horizontal running posture was re-assumed in a leveling-off stage. The action of the middle legs was redirected by rotations of the leg at the thoracal-coxal and the trochanteral-femoral joints. The subsequent extension movements of the coxal-trochanteral and femoral-tibial joints were within the range seen during horizontal running. The structure of proximal leg joints allows for flexibility in leg use by generating subtle, but effective changes in the direction of leg movement. This architecture, along with the resulting re-direction of movements, provides a range of strategies for both animals and walking machines.
\end{abstract}

Keywords Climbing - Center of mass - Body-substrate angle $\cdot$ Kinematics $\cdot$ Joint angle

\footnotetext{
J.T. Watson · R.E. Ritzmann $(\bowtie) \cdot$ A.J. Pollack

Department of Biology,

Case Western Reserve University,

10900 Euclid Avenue, Cleveland,

OH 44106-7080, USA

E-mail: rer3@po.cwru.edu

Tel.: + 1-216-3683554

Fax: + 1-216-3684672

S.N. Zill

Department of Anatomy,

Cell and Neurobiology,

Marshall University,

Huntington, WV 25704, USA
}

\begin{abstract}
Abbreviations $C o M$ center of mass $\cdot C T r$ coxatrochanter joint $\cdot F T i$ femur-tibia joint $\cdot T_{1}$ first thoracic (prothoracic) segment or leg $\cdot T_{2}$ second thoracic (mesothoracic) segment or leg $\cdot T_{3}$ third thoracic (metathoracic) segment or leg $\cdot \operatorname{ThC}$ thorax-coxa joint $\cdot \operatorname{Tr} F$ trochanter-femur joint
\end{abstract}

\section{Introduction}

Terrestrial locomotion using jointed legs is a complex adaptive behavior. Current understanding of walking and running is based mainly on studies that have recorded kinematic and/or force data from animals on smooth horizontal surfaces (Blickhan and Full 1993; Pearson 1993; Watson and Ritzmann 1998). However, a remarkable quality of legged animals is their agility in traversing diverse terrains. Legged animals readily clamber over obstacles, run on all manners of slopes, pick their way over discontinuous surfaces, and compensate for surface irregularities. The present studies were initiated to examine the specific changes in leg movements that occur as cockroaches of the species Blaberus discoidalis, adapt their locomotion to climb over barriers. These alterations provide insight to the mechanisms of control of insect walking and can also indicate effective strategies for limb use in legged robots.

Previous studies have shown that insects walking on a horizontal flat surface use a metachronal gait, in which the legs on each side step in a wave moving from back to front (Delcomyn 1971; Greene and Spirito 1979). Insects increase step frequency primarily by increasing the speed of leg extension during stance phase (Pearson 1976). As the duration of leg retraction decreases relative to protraction, the gait pattern shifts to an alternating tripod gait in which the front and hind legs on one side move as a unit along with the middle leg on the opposite side. This statically stable unit alternates with the tripod formed by the remaining three legs (Hughes 1952; Wilson 1966).

Although the tarsi (feet) of the legs making up a tripod move in synchrony, data on ground reaction 
forces as well as electromyograms combined with kinematic analysis clearly demonstrate that each pair of legs plays a unique role in supporting and moving the animal (Full et al. 1991; Watson and Ritzmann 1998). Typically, the rear legs generate propulsive forces that produce much of the forward motion. To generate forces along the long axis of the animal's body, the two principle distal joints, the coxa-trochanter joint $(\mathrm{CTr})$ and the femur-tibia (FTi) joints extend in near synchrony (Watson and Ritzmann 1998). In the middle legs, the homologous joints also move synchronously, but the excursion of the FTi joint is smaller than that of the $\mathrm{CTr}$ joint. The location of the middle leg results in a movement that produces a biphasic ground reaction force consisting of a braking phase followed by an accelerating phase (Full et al. 1991). In the front legs there are large movements at the proximal thoracal-coxal joint(s), which have three degrees of freedom (Tryba and Ritzmann 2000) and the CTr and FTi joints do not always move in strict synchrony. Rather, these legs act like arms reaching forward and to the side to explore the surrounding environment while providing braking ground reaction forces (Full et al. 1991).

In order to climb over substantial obstacles, an animal must ultimately raise its center of mass $(\mathrm{CoM})$ to the height of the obstacle. Most insects run with the body nearly parallel to the ground (Cruse 1976), so climbing over an obstacle might also involve tilting the front of the body upwards to grasp the face or edge with the legs. An insect that encounters an obstacle as it is walking could either climb over it by making minor modifications of the tripod gait, or it could change to a very different set of leg movements. Our data suggest that the deathhead cockroach surmounts obstacles using relatively subtle changes in leg orientation and body posture.

\section{Materials and methods}

Animals

Cockroaches (B. discoidalis) were housed in 20-1 plastic buckets, half filled with aspen shavings, and were held at $27^{\circ} \mathrm{C}$ in a $12 \mathrm{~h}$ light:12 h dark circadian cycle. Commercial dry chicken starter and water were provided ad libitum. We used only intact, adult female cockroaches: length of the insects used was $46.1 \pm 2.0 \mathrm{~mm}$ (mean $\pm \mathrm{SD}$ ) and the body weight was $3.31 \pm 0.42 \mathrm{~g}$.

Preparation and apparatus

We highlighted the ventral surface of the thoracal-coxal (ThC) joint, CTr joint, the anterior and lateral surfaces of the tibia just distal to the FTi joint and just proximal to the tibial-tarsal joint with white paint to facilitate visualizing them against the dark thorax of the cockroach. We placed each cockroach on a treadmill that allowed us to observe the animals during climbing and horizontal running. A clear acrylic chamber positioned just above the treadmill belt constrained the cockroach to run on the belt in an area of $5 \mathrm{~cm} \times 17 \mathrm{~cm}$. The $5 \mathrm{~cm}$ width of the belt was approximately 1.2 times body length and 1.5 times the span of the (middle) tarsi during running; which allowed unhindered running parallel to the belt motion. The treadmill ran at a constant speed during bouts of horizontal running. However, for climbing it was only moved to position cockroaches in front of the camera prior to the climbing movements. Thus, the animal was not subjected to acceleration due to the treadmill changing speed during the recoding period. The treadmill apparatus is depicted in Fig. 1a.

Cockroaches climbed over clear acrylic blocks of $50 \mathrm{~mm}$ length $\times 45 \mathrm{~mm}$ width $\times 5.5 \mathrm{~mm}$ height placed on the treadmill belt with one edge perpendicular to the direction of the insect's motion. A higher barrier was constructed by placing a second block on top of the first for an overall height of $11 \mathrm{~mm}$. As we will show, the choice of block heights was instructive, because each required the animal to use a different climbing strategy.

\section{Kinematics}

In order to monitor joint movement in three dimensions we recorded two orthogonal views. We recorded video images of the lateral view of the cockroach through the acrylic chamber and the ventral view through the acetate belt via a $19 \mathrm{~cm} \times 7 \mathrm{~cm}$ mirror mounted at a $45^{\circ}$ angle to the belt and immediately below it Climbing movements were recorded at 250 frames $\mathrm{s}^{-1}$ with a single camera Redlake digital video system that either downloaded the images to videotape for storage and analysis or stored them directly to digital files on a hard drive. We captured individual fields from videotaped data using a frame grabber card on a PC. Positions of various objects were digitized using the computer's mouse in conjunction with motion analysis software (MotionTV, DataCrunch Systems, Calif., or MA Studio, Graftek Imaging, Austin, Tex.). We digitized from each video field the ventral and lateral projections of the ThC, CTr, FTi, and the tibial-tarsal joints of the legs of interest, as well as the anterior tip of the head and the posterior tip of the abdomen, (Fig. 1b, c). We then calculated the true ThC, CTr and FTi joint angles in three-dimensional space from the ventral and lateral projected images (Marx et al. 1993). We defined the second abdominal (A2) segment as the center of mass of the cockroach (Nelson et al. 1997). The $x$-, $y$ - and $z$-planes of the animal were defined as shown in Fig. $1 \mathrm{~b}$.

\section{Data analysis}

We smoothed the data on joint angles using an even-weighted, moving-average of three data points. Criteria for definition of the phases of leg movements were the same as described previously (Watson and Ritzmann 1998). We compared kinematic parameters between different stages of the climbing sequence (see below) with one-way analysis of variance followed by pair-wise comparisons with $t$-tests or rank-sum tests when appropriate.

\section{Results}

An insect that encounters an obstacle as it is walking could do one of three things. First, it could climb over the obstacle by making little or no modifications of the tripod gait. Second, it could change to a completely different set of leg movements. Third, it could use normal walking movements, coupled with postural adjustments to direct the movement of its body over the obstacle. To assess what actually happens, we observed the changes in body attitude and specific leg movements made by animals as they climbed over the two different sized blocks described above.

\section{Changes in body attitude associated with climbing}

We measured the changes in CoM and body-substrate angle that occurred during stance phase of steps 
Fig. 1 a The experimental setup for high-speed videography of cockroaches on a motorized treadmill (note: animal not to scale with treadmill). b Blaberus discoidalis lateral and ventral views and conventions for defining $x$-, $y$-, and $z$-planes. Dimensions in $\mathbf{b}$ indicate scale of the animal. c Leg of $B$. discoidalis, ventral showing all leg joints

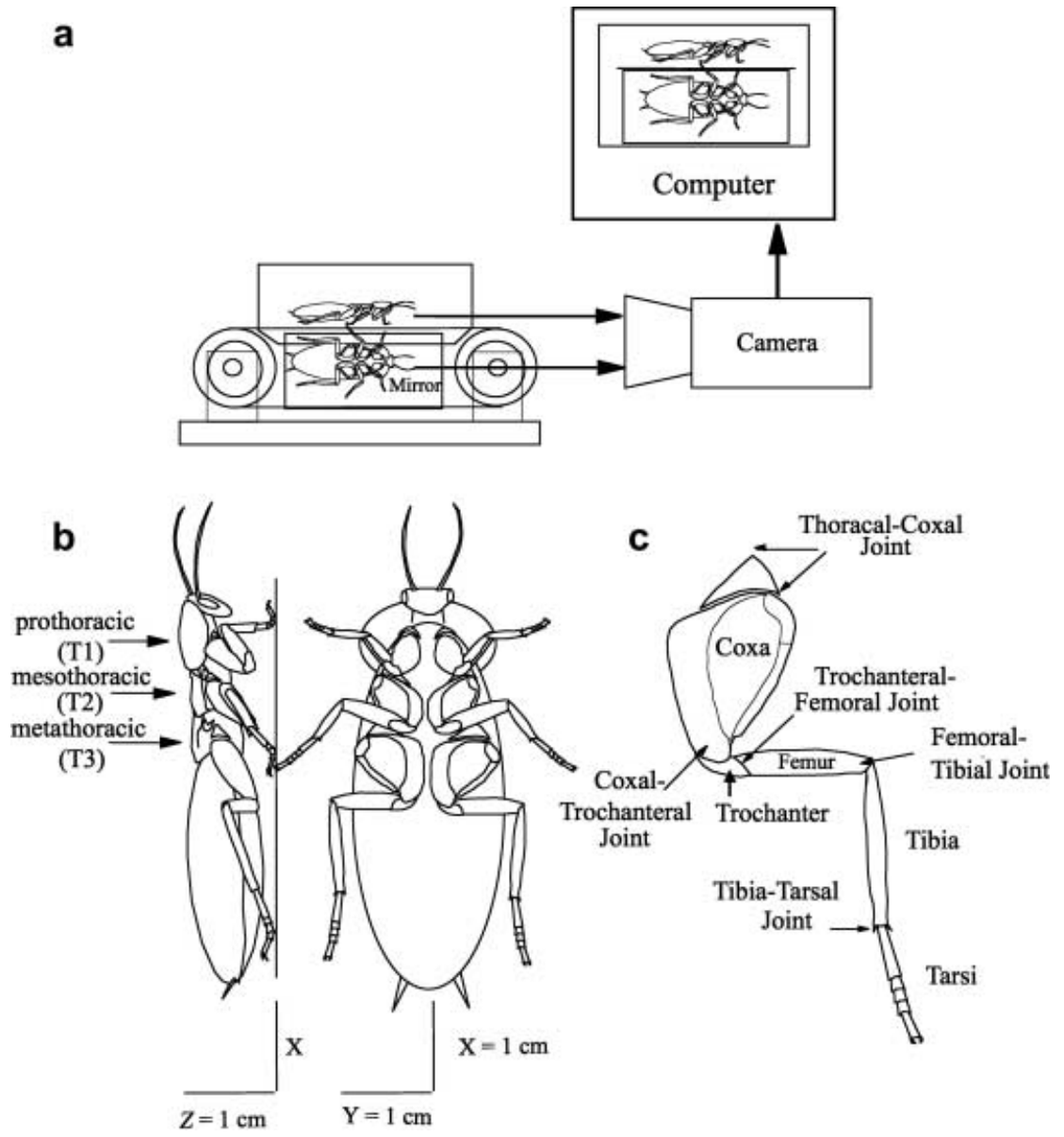

associated with each climb. The analysis included the period just preceding the step when contact was first made with the test obstacle through the point where the animal surmounted the block.

\section{5-mm obstacle climbs}

Figure 2 shows selective frames from climbs over 5.5$\mathrm{mm}$ and $11-\mathrm{mm}$ obstacles. Both show changes in bodysubstrate angle. However, the timing of these events demonstrates important differences. During horizontal running, the tarsi of the front legs are normally lifted higher than $6 \mathrm{~mm}$ (mean $\pm \mathrm{SEM}=6.8 \pm 0.4 \mathrm{~mm}$ ). Therefore, when the cockroach approaches a $5.5-\mathrm{mm}$ block, the front legs require no alteration in swing trajectory to reach the top of that obstacle (Fig. 2a). However, for 11-mm obstacles the front tarsus would encounter the vertical surface of the barrier well below the top (Fig. 2d, arrow).

Video images such as those shown in Fig. 2 suggest that the animal's body-substrate angle changes after the front leg reaches a 5.5-mm block (Fig. 2b) but before reaching the top of an 11-mm block (Fig. 2e). That would imply that $B$. discoidalis alters posture in anticipation of higher blocks. In Fig. $3 \mathrm{~b}$ we plot the change in CoM against the change in body-substrate angle that occurs during the stance phase of four leg cycles, starting with the cycle immediately preceding the encounter with the block and proceeding through the point where all of the animal's legs are on the top of the block. During horizontal running, body-substrate angle and $\mathrm{CoM}$ have only a slight decrease in values between the beginning and end of the stance (data not shown), reflecting the up and down body motions that occur during walking (Full and Tu 1991). The data for the step just prior to reaching the $5.5-\mathrm{mm}$ obstacle (step -1) reflect that pattern (Fig. 3b). The animal is tilted up only after the front leg is placed upon the top of the block during step 0 , confirming that the change in body substrate angle is a consequence of the block rather than an adjustment in anticipation of it. With this increase in body-substrate angle, extension of the legs during step +1 propels the animal up to the top of the block as indicated by the increase in CoM. The body levels off in step +2 as seen by the predominantly negative change in body-substrate angle.

\section{Change in strategy for 11-mm obstacles}

During horizontal running the front legs do not typically lift high enough to reach the top of an 11-mm obstacle. Nonetheless, cockroaches usually placed their front legs 


\section{$5.5 \mathrm{~mm}$ obstacle climb}

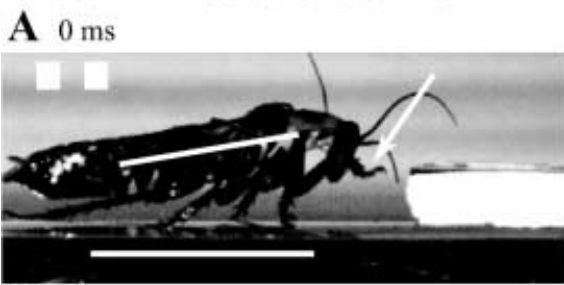

B $44 \mathrm{~ms}$

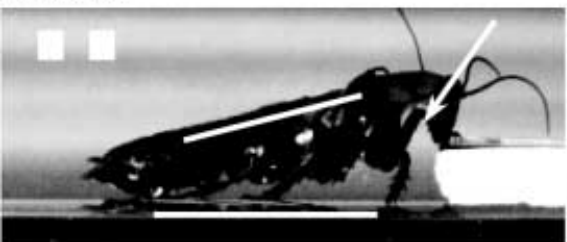

C $64 \mathrm{~ms}$

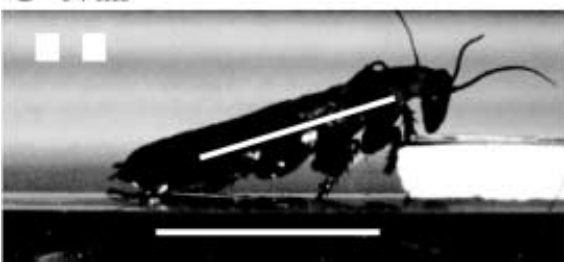

Fig. 2a-f Body adjustments necessary for climbing over obstacles. a-c $B$. discoidalis approaching and starting a climb over a $5.5-\mathrm{mm}$ block. Note that the front leg swing movement places the tarsus on top of the $5.5-\mathrm{mm}$ block with no additional adjustments of the body angle, and the body angle increases after the tarsus is placed on top of the block in B. $\mathbf{d}-\mathbf{f}$ B. discoidalis approaching and starting a climb over the $11-\mathrm{mm}$ obstacle. Note that the body angle increases before the tarsus of the front leg touches the top of the $11-\mathrm{mm}$ obstacles. The arrows in e point to the right front tarsus suspended above the block and the rotated middle leg indicating that the change in body angle is generated by middle leg extension. The front leg contacts the top of the block between $\mathbf{e}$ and $\mathbf{f}$. Time indicated above individual pictures indicates time at which frame occurred relative to the first frame (a and $\mathbf{d})$. Note that the animal paused for approximately $2 \mathrm{~s}$ prior to initiating the climb in $\mathbf{e}$

directly on top of the 11-mm block without performing searching movements as seen in locusts (Pearson and Franklin 1984). In $65 \%$ of the trials the front leg did not touch the front of the block and in the remaining $35 \%$ the front leg contacted the face of the block, but did not really search for the top edge. $B$. discoidalis accomplishes this by a change in strategy when climbing over $11-\mathrm{mm}$ blocks (Figs. 2d-f, 3). Here the typical running pattern is seen for the leg cycle that occurs two cycles before reaching the obstacle (step -2 , see Fig. 3c, note similarity to step -1 in Fig. 3b). However, the increase in bodysubstrate angle is now shifted to step -1 , where the animal is immediately in front of the barrier. That is, before the front legs actually make contact with the obstacle, the animal has already tilted its body upward so that the front legs can reach the top of the block. The change in body-substrate angle during step -1 is sometimes accompanied by an increase in CoM height. In the next leg cycle (step 0 , or the step in which the front leg first contacts the top of the block), there is usually a further

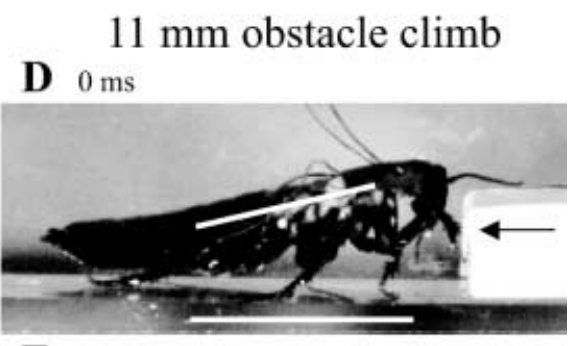

E $2114 \mathrm{~ms}$

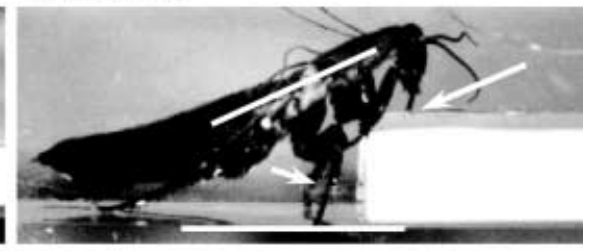

F $2560 \mathrm{~ms}$

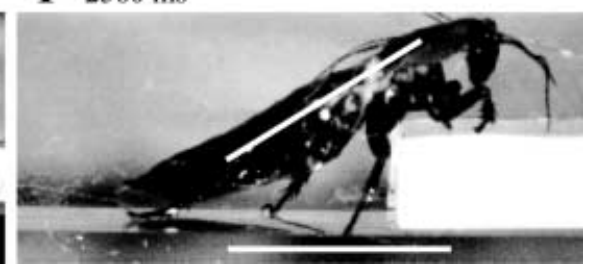

raising of the CoM with little additional change in body substrate angle. Finally, in step +1 , the animal places all its legs on the top of the obstacle and levels off, generating a decrease in body substrate angle while $\mathrm{CoM}$ height continues to increase.

The data described in Fig. 3c allow us to kinematically define two stages associated with the majority of $11-\mathrm{mm}$ climbs. The rearing stage generates the change in body-substrate angle before any leg reaches the block. This stage may or may not be associated with a change in CoM. However, by definition it always involves an increase in body-substrate angle. In the subsequent rising stage, the animal's CoM is raised upward with little or no further change in body-substrate angle.

Although the description given above (based upon the data in Fig. 3c) was the most common strategy for climbing over large barriers, $B$. discoidalis could utilize at least four other strategies in ascending the $11-\mathrm{mm}$ obstacle (Fig. 4). In very few cases the animal extended both hind legs in a jumping movement (not shown in Fig. 4b). Two other rare strategies involved changes in CoM without accompanying changes in body substrate angle. These were (1) simultaneously extending all six legs to produce an elevation of the whole body (labeled "Elevate"), and (2) using an unusually high swing trajectory for one front leg in order to place it on top of the block while keeping the body horizontal. In the latter case, the animal could then push down on the block with its front leg, essentially using the strategy employed to climb over smaller barriers. The predominant strategy ("Rear up") was to change the body angle before placing the front legs on the block (during step -1 in Fig. 3). This was most commonly accomplished by rearing the front of the body upwards prior to contacting the 


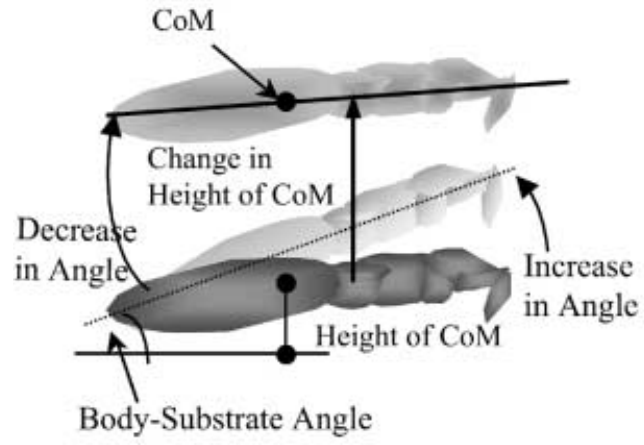

B Change in Body-Substrate Angle \& CoM Height During Extension Phase: $5.5 \mathrm{~mm}$ Climb

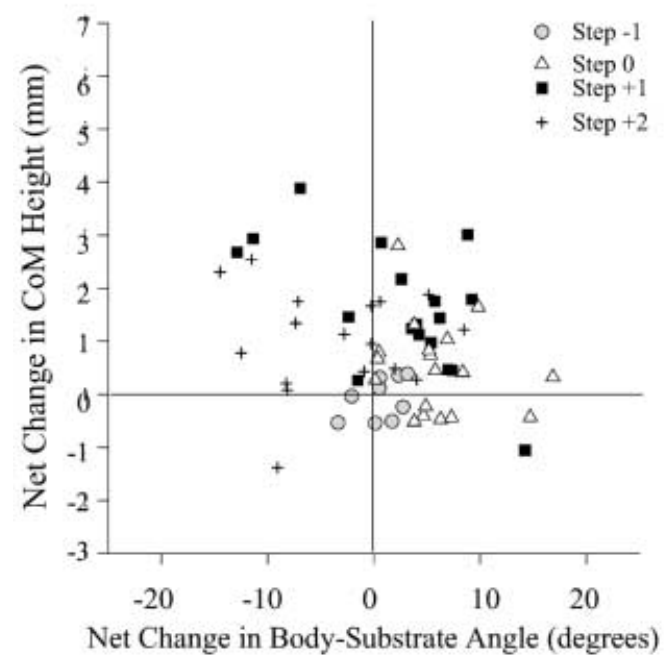

C Change in Body-Substrate Angle \& CoM Height During Extension Phase: $11 \mathrm{~mm}$ Climb

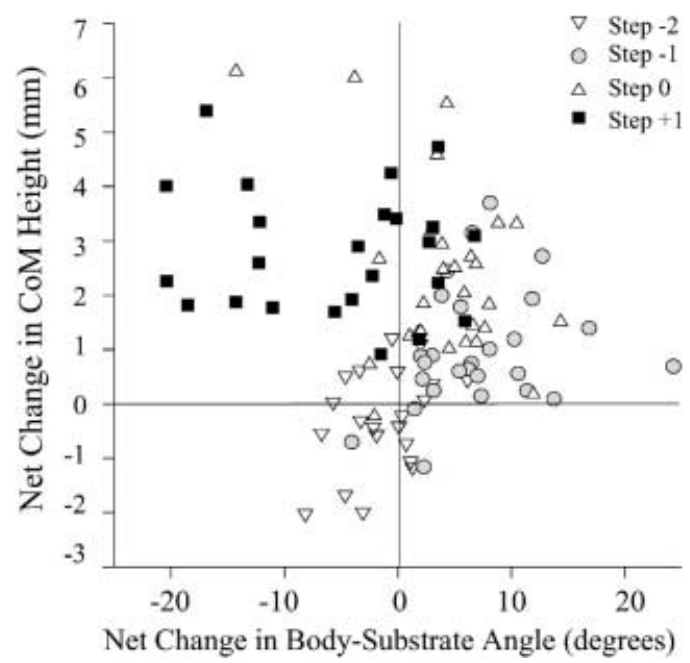

Fig. 3a,b Net change in body-substrate angle and height of center of mass in B. discoidalis approaching and climbing over rectangular acrylic blocks of varying height. Step 0 refers to the step in which either the head or one first thoracic (prothoracic) $\left(\mathrm{T}_{1}\right)$ leg contacts the top of the block. a Explanation of terms. b Plot of net change in center of mass (CoM) during each step cycle versus net change in body-substrate angle during the same step cycle for $5.5-\mathrm{mm}$ obstacle climbs: Note the increase in body angle occurs first in step 0 when a $T_{1}$ leg contacts the top of the block. c Similar plot for $11-\mathrm{mm}$ obstacle climbs. Note the increase in body substrate angle occurs in step -1 , before any $\mathrm{T}_{1}$ leg contacts the top of the block

block, while all legs remained on the substrate. On few occasions animals simply butt the head ("Head butt") against the block thereby forcing the body upward.

Gait changes associated with climbing

The pattern of inter-leg coordination used during climbing over large obstacles was distinct from that seen in horizontal running as well as that seen in climbing over $5.5-\mathrm{mm}$ obstacles. In the tripod gait typically used during horizontal running, each leg protracts out of phase with both the contralateral leg in the same segment and ipsilateral legs in adjacent segments. Phase can be represented as a number between 0 and 1, perfect anti-phase would be a phase of 0.5 . Phase relationships for running and climbing are summarized in Fig. 5. In horizontal running, the mean phase between pairs of legs in each thoracic segment was close to 0.5 , as was the mean phase of adjacent ipsilateral legs (Fig. 5a, d). During climbing over 5.5-mm obstacles, the phase relationships of each pair of legs were not significantly altered from those seen during running (see Fig. $5 \mathrm{~b}, \mathrm{~d}$ ). However, when $B$. discoidalis climbed over the $11-\mathrm{mm}$ obstacles, changes were seen. The animals frequently performed extra short steps (i.e., one leg performed more swing phases than its contralateral partner did) as the body reared up and the animal sought to place its tarsi closer to the obstacle. Such extra steps were seen in the front legs in all climbs, in middle legs in 7 of 12 climbs and in the hind legs in 5 of 12 climbs. In addition, when the extra steps were factored out, the middle and hind legs protracted more in phase with their contralateral partners.

Phase data from several events are summarized in Fig. 5d. For both horizontal running and $5.5-\mathrm{mm}$ obstacle climbs, intra-segmental phases remained near 0.5 indicating a typical tripod gait. However, in 11-mm climbs, there was a significant shift away from a phase of 0.5 during rearing for all leg pairs and for rising for the second thoracic (mesothoracic) $\left(\mathrm{T}_{2}\right)$ leg pair, suggesting an increase in periods of dual support. This pattern of dual support may assist the animal as it moves itself upward against gravity.

It should be noted that during climbs over large obstacles the animal often slows down. Thus, it is possible that the changes seen here are due to a decrease in speed rather than to an altered climbing strategy. Data 


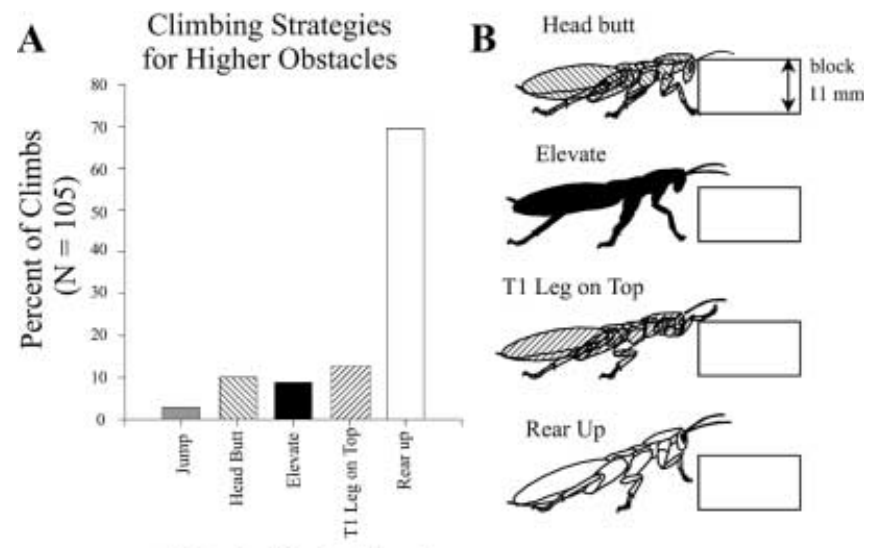

Behavior During Step - 1

Fig. 4a,b Climbing strategies used by $B$. discoidalis to surmount $11-\mathrm{mm}$ obstacles. a Frequency of behaviors $B$. discoidalis performed in the step preceding contact with the top of the 11-mm obstacle. b Illustration of each of the behaviors except the jumping strategy

reported previously (Delcomyn 1971) argue against this explanation. During walking, even at $1.5 \mathrm{~Hz}$, the contralateral legs still alternate during protraction and phase remains close to 0.5 . The climbing movements shown in Fig. 5c occurred at $2.29 \mathrm{~Hz}$ and the movements for the entire population of animals summarized in Fig. 5 d occurred at $2.57 \pm 0.23 \mathrm{~Hz}$.

How are postural changes accomplished during rearing and climbing?

The rearing stage of the $11-\mathrm{mm}$ climb requires $B$. discoidalis to elevate the front of its body so that it can place the front legs within reach of the top of the obstacle. It can accomplish this task in one of three ways: (1) execute a dorsal flexion of the body, (2) change the excursion of leg joints, or (3) change the orientation of various legs. These possibilities are not mutually exclusive.

\section{Dorsal flexion}

Cockroaches can flex each body segment and during climbing we have noted particularly large ventral flexions in thoracic segments, particularly between the proand mesothoracic segments, that may contribute to keeping the legs in a mechanically efficient posture (e.g., Figs. 2e, f, 7c). A simple solution for rearing would be to flex the body dorsally around these segments so that the front legs could reach the top of the block. However, of nine animals examined for body flexion, only four showed any dorsal flexion of the body, and then in only 8 of 71 climbs. Even in these cases, the dorsal flexion that occurred was insufficient to complete the climb. Therefore, in the majority of rearing movements, in which the body-substrate angle increased, dorsal flexion of the body was not a major factor.



D Phase Relationships among Legs during Running and Climbing

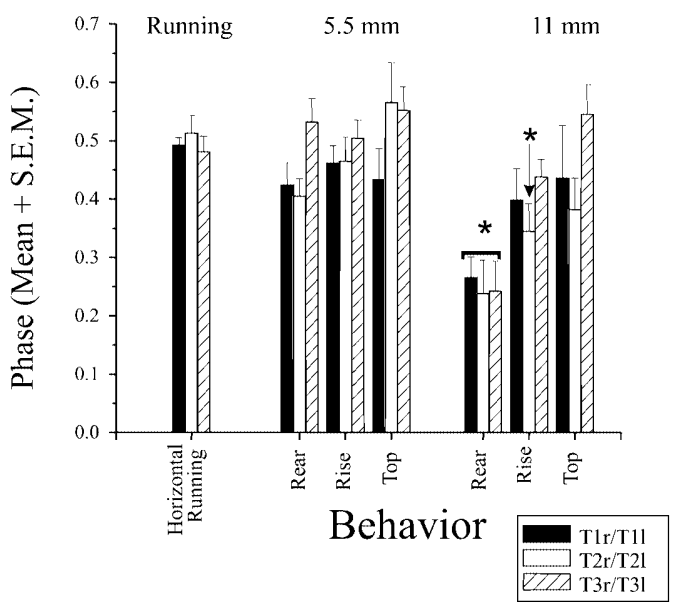

Fig. 5 Gait patterns during a horizontal running, b climbing over a 5.5-mm obstacle, and c climbing over an 11- $\mathrm{mm}$ obstacle. In each figure the bars indicate the swing phase (leg not contacting substrate) of the respective leg. The arrows in b c indicate when each leg first contacted the top of the obstacle. d Phase relationships among legs during horizontal running and climbs onto 5.5-mm obstacles and 11-mm obstacles. An asterisk Indicates value significantly different from the corresponding value for horizontal running. Note that for $11-\mathrm{mm}$ rearing all leg pairs had significantly lower phase numbers from the typical 0.5 of horizontal running, while for rising only the $T_{2 r} / T_{21}$ phase number was significantly different

\section{Change in joint excursion}

During running the joints that make the most effective movements in the middle and hind legs are the CTr and FTi joints. Rearing and rising movements might require increases in the excursion of one or more of those joints.

Not surprisingly, as the animal climbs over $5.5-\mathrm{mm}$ objects the kinematics of these joints are indistinguishable from data taken during running (data not shown). There were no significant differences in joint angle extremes, joint excursions, or joint velocities, compared to horizontal treadmill running.

As the animal climbs over the larger 11-mm obstacles, there are still no significant changes in CTr or FTi joint excursion for the middle legs during either rearing or rising stages (Table 1; Fig. 6a). The CTr joint moves to the same maximum and minimum angles as seen during horizontal running (Table 1). Even though the FTi joint makes the same excursion, the maximum and minimum angles are significantly reduced in virtually all 
Table 1 Kinematic data for middle $\left(\mathrm{T}_{2}\right)$ leg trochanter joint $(\mathrm{CTr})$ and femur-tibia joint (FTi) joints during climbing over $11-\mathrm{mm}$ blocks. Data are presented as mean \pm SEM. Pair-wise shows which comparisons were significant using pair-wise test following oneway ANOVA ( $C$ coxa, $T r$ trochanter, $F$ femur, $T i$ tibia, $N S$ not significant)

\begin{tabular}{|c|c|c|c|c|c|}
\hline Middle leg & Running & Rearing & Rising & Leveling & Pair-wise \\
\hline$n$ for $\mathrm{C} \operatorname{Tr}$ joint & 26 & 15 & 13 & 14 & \\
\hline $\begin{array}{l}\text { Mean CTr velocity during } \\
\text { extension (degrees } \mathrm{s}^{-1} \text { ) }\end{array}$ & $337.6 \pm 23.4$ & $154.5 \pm 26.4$ & $169.5 \pm 33$ & $125 \pm 11.7$ & Run $>$ all \\
\hline Minimum CTr joint angle (degrees) & $24 \pm 1.5$ & $28.1 \pm 2.3$ & $30.6 \pm 2.4$ & $31.1 \pm 2$ & NS \\
\hline Maximum CTr joint angle (degrees) & $69.3 \pm 0.9$ & $70.4 \pm 4.6$ & $71.6 \pm 3.6$ & $69.7 \pm 3.3$ & NS \\
\hline CTr excursion (degrees) & $45.3 \pm 1.9$ & $42.3 \pm 6.2$ & $41 \pm 4.4$ & $38.6 \pm 4$ & NS \\
\hline $\mathrm{CTr}$ extension duration (ms) & $160.6 \pm 8.8$ & $309.7 \pm 38.1$ & $354.6 \pm 89.3$ & $378 \pm 74.8$ & All $>$ run \\
\hline Body-substrate angle change (degrees) & $-1.6 \pm 0.8$ & $7.6 \pm 2.1$ & $3.7 \pm 1.9$ & $-3 \pm 0.3$ & $\begin{array}{l}\text { Rear }>\text { run, level; } \\
\text { rise }>\text { run }\end{array}$ \\
\hline $\begin{array}{l}\text { Body-height change }(\mathrm{mm}) \\
n \text { for FTi }\end{array}$ & $\begin{array}{l}-0.1 \pm 0.2 \\
26\end{array}$ & $1.3 \pm 0.6$ & $\begin{array}{l}5.5 \pm 4.3 \\
13\end{array}$ & $14.7 \pm 0.4$ & Rise, level > run \\
\hline $\begin{array}{l}\text { Mean FTi velocity during } \\
\text { extension (degrees } \mathrm{s}^{-1} \text { ) }\end{array}$ & $279.5 \pm 27.6$ & $153.2 \pm 30.2$ & $143.3 \pm 29.2$ & $102.1 \pm 13.8$ & Run $>$ all \\
\hline Minimum FTi joint angle (degrees) & $91.2 \pm 2.6$ & $71.4 \pm 6.3$ & $69.1 \pm 6.1$ & $65 \pm 5.6$ & Run $>$ all \\
\hline Maximum FTi joint angle (degrees) & $123.7 \pm 2.6$ & $107.4 \pm 6.3$ & $103.8 \pm 4.2$ & $99.6 \pm 3.7$ & Run $>$ level, rise \\
\hline FTi excursion (degrees) & $32.4 \pm 2$ & $36 \pm 7.6$ & $34.7 \pm 4.3$ & $34.5 \pm 4.8$ & NS \\
\hline FTi extension duration (ms) & $164.2 \pm 11.2$ & $326.1 \pm 46.9$ & $338.7 \pm 97.5$ & $360.3 \pm 79.9$ & All $>$ run \\
\hline
\end{tabular}

climbing stages. In the hind legs (Fig. 6b), the only significant change from running is a decrease in CTr excursion during rearing (Table 2). This alteration may reflect a decrease in forward motion during the rearing stage of the climb.

The cockroaches usually slowed their locomotion to surmount the 11-mm obstacle. For both the middle and hind legs, the durations of CTr and FTi joint extension were significantly greater and mean CTr and FTi joint velocities were significantly lower in middle and hind legs during most stages of $11 \mathrm{~mm}$ block-climbing than the average found in horizontal running (Tables 1,2 ).

\section{Change in leg orientation}

The rearing stage could be accomplished by changing the orientation of one or more legs so that subsequent $\mathrm{CTr}$ and FTi extension, similar to that used during running, would push the animal upward rather than forward. For example, a change in orientation of one or both middle legs so that the tibia now approaches an angle perpendicular to the ground would be sufficient to rear the front of the animal upward even with normal distal joint excursions.

In $11-\mathrm{mm}$ obstacle climbs, $88 \%$ of rearing movements are associated with a rotation of the middle leg relative to the body, resulting in a similar change relative to the substrate, either before or during the change in body-substrate angle (Fig. 7a). Changes in middle leg orientation are particularly evident in the lateral projection of the angle between the tibia and the body axis, i.e., the $z$-plane angle of the tibia (Fig. 2e, arrow). We will refer to this change in orientation as a tibial rotation. Note the rotation of the middle leg changes the orientation of the tibia relative to the body (body/tibia angle: running $=37.2 \pm 2.8$, rearing $=56.8 \pm 4 ; n=7$ animals, $P<0.0002)$ as well as the substrate and typically begins during swing. Thus, it is an active postural change not merely a passive consequence of change in the orientation of the body relative to the substrate. In $53 \%$ of the climbs, the middle leg rotated prior to the rearing stage (Fig. 7a). However, in $35 \%$ of the climbs, tibial rotation of the middle leg occurred after rearing began. In the latter cases the initial rearing appeared to occur as a result of front leg movements. Even in these climbs, the middle leg then rotated to provide a larger base of support as the front leg proceeded to the top of the block. Middle leg extension then completed the rearing movement.

Tibial rotations are also seen in the middle legs during subsequent climbing movements, but these occur less often than during rearing. In contrast, a similar change in tibia angle is seen in the hind leg during the rising movement, but to a lesser extent during rearing (Fig. 7b, c). These adjustments allow the hind legs to maintain contact with the block or the substrate during the rising movements. Both legs also show tibial rotations during leveling off at the top of the obstacle.

In most normal climbs it is likely that both front and middle leg actions contribute to rearing. Can we differentiate the relative importance of the front and middle legs during rearing movements? In $15 \%$ of rearing movements, both front legs were in swing phase and could not, therefore, generate any ground reaction forces. Certainly in those cases the middle legs generated rearing on their own. To further test the individual contributions of front and middle legs to the rearing movements, we amputated either both front legs (Fig. 8a, b) or both middle legs (Fig. 8c, d) at the level of the trochanter-femur joint (TrF) joint (i.e., the autotomy plane). Front leg amputees could still negotiate both $5.5-\mathrm{mm}$ blocks (10 animals, 80 attempts, all successful) and 11-mm blocks (12 animals, 92 attempts, all successful) using rearing and rising stages. However, in many instances rearing was exaggerated (Fig. 8b). 
Fig. 6 Kinematics of mesothoracic $\left(\mathrm{T}_{2}\right)$ leg (a )and metathoracic $\left(\mathrm{T}_{3}\right)$ leg (b) while climbing $11 \mathrm{~mm}$ obstacles. Coxa-trochanter $(\mathrm{CTr})$ and femur-tibia (FTi) joint angle records from each leg synchronized with body substrate angle and CoM height. "Middle Leg Tibial

Rotation" and "Hind Leg Tibial Rotation" arrows indicate when the distal segments of the digitized leg are re-oriented to facilitate climbing. Stages of the climb are indicated above the records
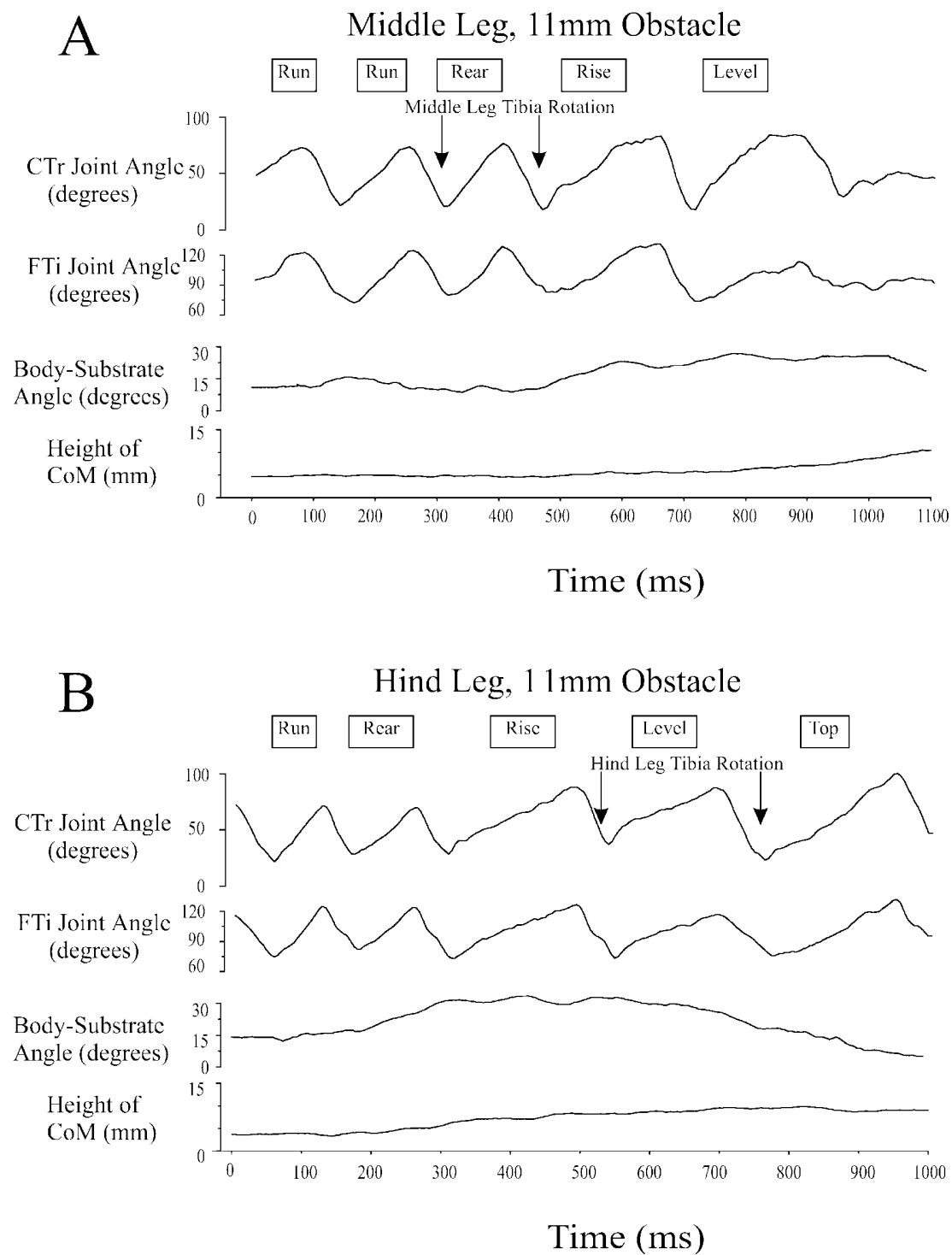

Middle leg amputees could also climb over both size obstacles $(5.5 \mathrm{~mm}: 8$ animals, 44 attempts, all successful; $11 \mathrm{~mm}: 8$ animals, 43 attempts, 41 successful). In these cases, the animals reared up on their front legs then placed one of the front tarsi on top of the block in a movement similar to the " $\mathrm{T}_{1}$ Leg on Top" strategy described in Fig. 4. Qualitatively, the middle leg amputees appeared to be clumsier at climbing tasks than front leg amputees. These observations suggest that although both pairs of legs are effective in executing the rearing stage of climbing, the middle legs play a more dominant role.

\section{Mechanism for changing leg orientation}

How does the animal rotate its leg in preparation for rearing? Since the CTr and FTi joints are unchanged from walking, movements of other joints must generate the tibial rotations during rearing and rising stages of the climb. The actions at the ThC and TrF joints can account for postural reorientation of the distal leg segments. The ThC is a complex joint with 3 degrees of freedom (Laurent and Richard 1986). The articulation with the greatest excursion ( $\alpha$-axis of Nelson et al. 1997, or P axis of Dresden and Nijenhuis 1953; Fig. 9a) produces ventral rotation of the coxa. In the middle leg, the $\alpha$-axis is oriented at approximately $45^{\circ}$ to the $x-y$ plane and $45^{\circ}$ to the $x-z$ plane. As a result, when the joint flexes, it rotates the entire leg outward and downward. Because this is a proximal joint, a small movement at the $\mathrm{ThC}$ joint results in a major rotation at the tibia. In side view, the action of the ThC joint appears to rotate the coxa around its long axis (Fig. 9b-c). However, a camera view positioned along the $\alpha$-axis reveals a simple $\alpha$ rotation (Fig. 9d-e).

Although the movement of this leg is obvious in a restrained animal as its leg is moved passively, it is less clear in the actual climbing videos. To determine if the proposed $\alpha$ rotation can account for the tibial rotation 
Table 2 Kinematic data for hind $\left(\mathrm{T}_{3}\right)$ leg CTr and FTi joints during climbing over 11-mm blocks. Data are presented as mean $\pm \mathrm{SEM}$. Pair-wise shows which comparisons were significant $(P<0.05)$ using pair-wise test following one-way ANOVA (NS not significant)

\begin{tabular}{|c|c|c|c|c|c|}
\hline Hind leg & $\begin{array}{l}\text { Horizontal } \\
\text { running }\end{array}$ & Rearing & Rising & Leveling & Pair-wise \\
\hline$n$ for $\mathrm{CTr}$ & 31 & 10 & 15 & 12 & \\
\hline $\begin{array}{l}\text { Mean CTr velocity during } \\
\text { extension }\left(\text { degrees } \mathrm{s}^{-1}\right)\end{array}$ & $280.3 \pm 16.8$ & $104.4 \pm 20$ & $173.2 \pm 25.6$ & $174.7 \pm 25.2$ & Run $>$ all \\
\hline Minimum CTr joint angle (degrees) & $21.2 \pm 0.8$ & $23.4 \pm 5.1$ & $19.2 \pm 1.7$ & $30.4 \pm 3.8$ & NS \\
\hline Maximum CTr joint angle (degrees) & $81 \pm 1.5$ & $67.3 \pm 4.8$ & $83.5 \pm 3.6$ & $86.5 \pm 5.8$ & Level $>$ rear \\
\hline $\mathrm{CTr}$ excursion (degrees) & $59.9 \pm 1.9$ & $44 \pm 5.2$ & $64.3 \pm 3.7$ & $56 \pm 5.9$ & Run, rise $>$ rear \\
\hline CTr extension duration (ms) & $235.8 \pm 17.2$ & $574.6 \pm 121.9$ & $481.4 \pm 57.9$ & $333.7 \pm 39.1$ & Rear, rise $>$ run \\
\hline Body-substrate angle change (degrees) & $-0.37 \pm 1.3$ & $6.77 \pm 0.97$ & $3.84 \pm 0.83$ & $-8.31 \pm 2.47$ & $\begin{array}{l}\text { Rear }>\text { level, run, } \\
\text { rise }>\text { level }\end{array}$ \\
\hline Body-height change (mm) & $-1.41 \pm 0.92$ & $1.03 \pm 0.26$ & $3.43 \pm 0.32$ & $2.86 \pm 0.39$ & $\begin{array}{l}\text { Rise }>\text { run, rear; } \\
\text { level }>\text { rear }\end{array}$ \\
\hline$n$ for $\mathrm{FTi}$ & 31 & 9 & 14 & 11 & \\
\hline $\begin{array}{l}\text { Mean FTi velocity during } \\
\text { extension }\left(\text { degrees } \mathrm{s}^{-1}\right)\end{array}$ & $291.3 \pm 23$ & $95.1 \pm 21$ & $157.7 \pm 26.7$ & $167.9 \pm 34.2$ & Run $>$ rear, rise \\
\hline Minimum FTi joint angle (degrees) & $67.2 \pm 0.9$ & $73.2 \pm 3.6$ & $67.3 \pm 1.7$ & $67.8 \pm 3.9$ & NS \\
\hline Maximum FTi joint angle (degrees) & $128 \pm 43$ & $116 \pm 7$ & $124.8 \pm 5.1$ & $115.2 \pm 6.4$ & NS \\
\hline FTi excursion (degrees) & $60.8 \pm 4.5$ & $42.8 \pm 7.6$ & $57.6 \pm 5.5$ & $47.4 \pm 8.6$ & NS \\
\hline FTi extension duration $(\mathrm{ms})$ & $238 \pm 14.3$ & $607.2 \pm 110.5$ & $464.6 \pm 61.1$ & $333.5 \pm 38.1$ & Rear, rise $>$ run \\
\hline
\end{tabular}

during climbing, we highlighted the cuticular ridge on the lateral edge of the coxa with a white line, so that we could follow its movement during rearing and compare that to the passive actions described above. We then measured the distance from the line on the coxa to a reproducible landmark on the thorax (the metathoracic spiracle) as we moved the leg passively around the $\alpha$-axis as described above (Fig. 9b, c) and noted how that distance increased (Fig. 9g). There was a similar increase in this distance during the rearing stage in an actively climbing animal when the tibia of the middle leg rotated (Fig. 9h), suggesting that the change in orientation of the middle leg could be largely accounted for by actions of this articulation. Although we cannot eliminate contributions from the other two degrees of freedom of the $\mathrm{ThC}$ joint, the most parsimonious explanation emphasizes the $\alpha$-axis.

The $\operatorname{TrF}$ joint also contributes to tibial rotation (Fig. 10a). This joint is often described as being fused. However, we observed a small but effective flexion movement at the $\operatorname{TrF}$ joint. The femur reductor muscle in the trochanter (Fig. 10b-d) actuates the joint, and stimulation of this muscle flexes it (Fig. 10b-e). Moreover, we have impaled two motor neurons that innervate the femur reductor muscle (Fig. 10f). When stimulated, these neurons also cause the femur to flex about the hinge towards the posterior aspect of the leg and, when the leg is in its typical posture, toward the dorsal aspect of the body. There is no antagonistic muscle for this joint. Thus, the return extension movement is apparently produced by passive joint properties. The TrF joint is oriented approximately $45^{\circ}$ to the long axis of the leg, so flexion results in a rotation of the tibia to an angle closer to perpendicular relative to both the body and the substrate. As with the ThC joint, the proximal location of the TrF joint means that a small movement at the joint produces a large excursion of the tarsus.

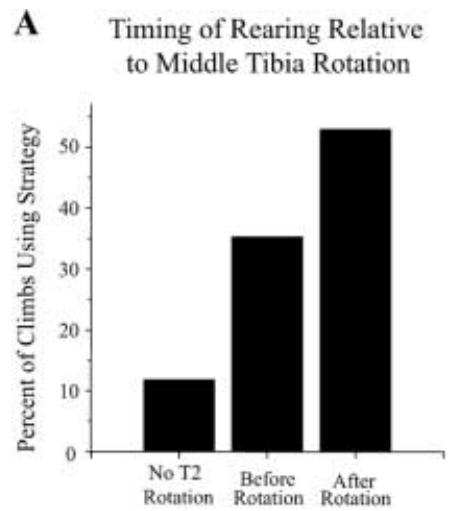

B Occurrence of Hind Tibia Rotation During Climbing
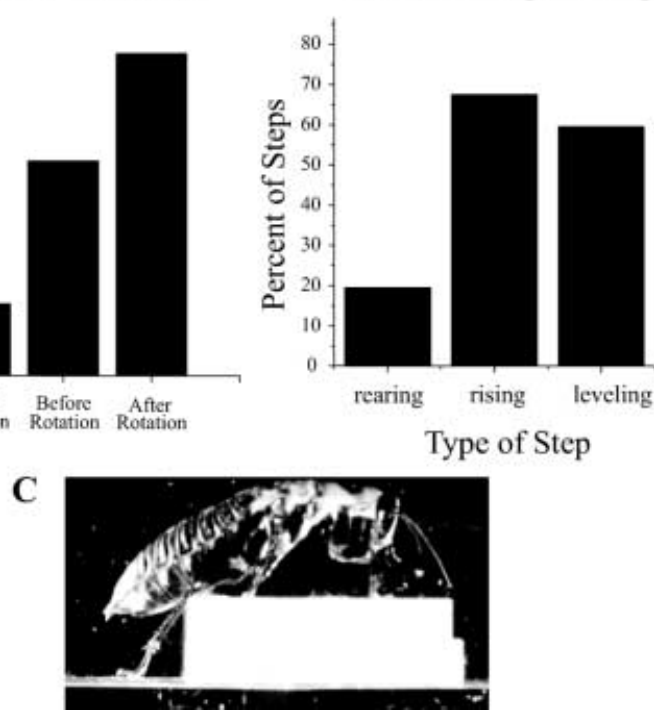

Fig. 7a-c Rearing and rising strategies involve postural adjustments in middle and hind legs. a Timing of the change in bodysubstrate angle relative to the postural adjustment of the middle leg (tibial rotation) during the rearing up behavior as the animal approaches the 11-mm obstacle. b Percentage of rearing, rising, and leveling steps in 11-mm climbs where $B$. discoidalis perform a tibial rotation of the hind leg. c Picture from a video record showing B. discoidalis surmounting an 11-mm obstacle using hind leg rotation. Compare the angle of the hind leg in this picture to normal orientation during running or at the outset of climbing as seen in Fig. 2

Thus, the $\operatorname{TrF}$ joint is capable of rotating the tibia. Does it contribute significantly to the tibial rotation that occurs during the rearing stage of a climb? To test this, we attempted to prevent its movement during climbing. This is a difficult joint to immobilize, because of its relatively small movement. However, we successfully immobilized it in the middle leg of three animals and the 
Fig. 8a-d Cockroaches can climb using only two pairs of legs. a, b Behavioral sequence showing a cockroach with the front legs amputated climbing a 5.5- $\mathrm{mm}$ obstacle (a) and an 11-mm obstacle (b). c, d Behavioral sequence showing a cockroach with the middle legs amputated climbing a $5.5-\mathrm{mm}$ obstacle (c) and an 11-mm obstacle (d)
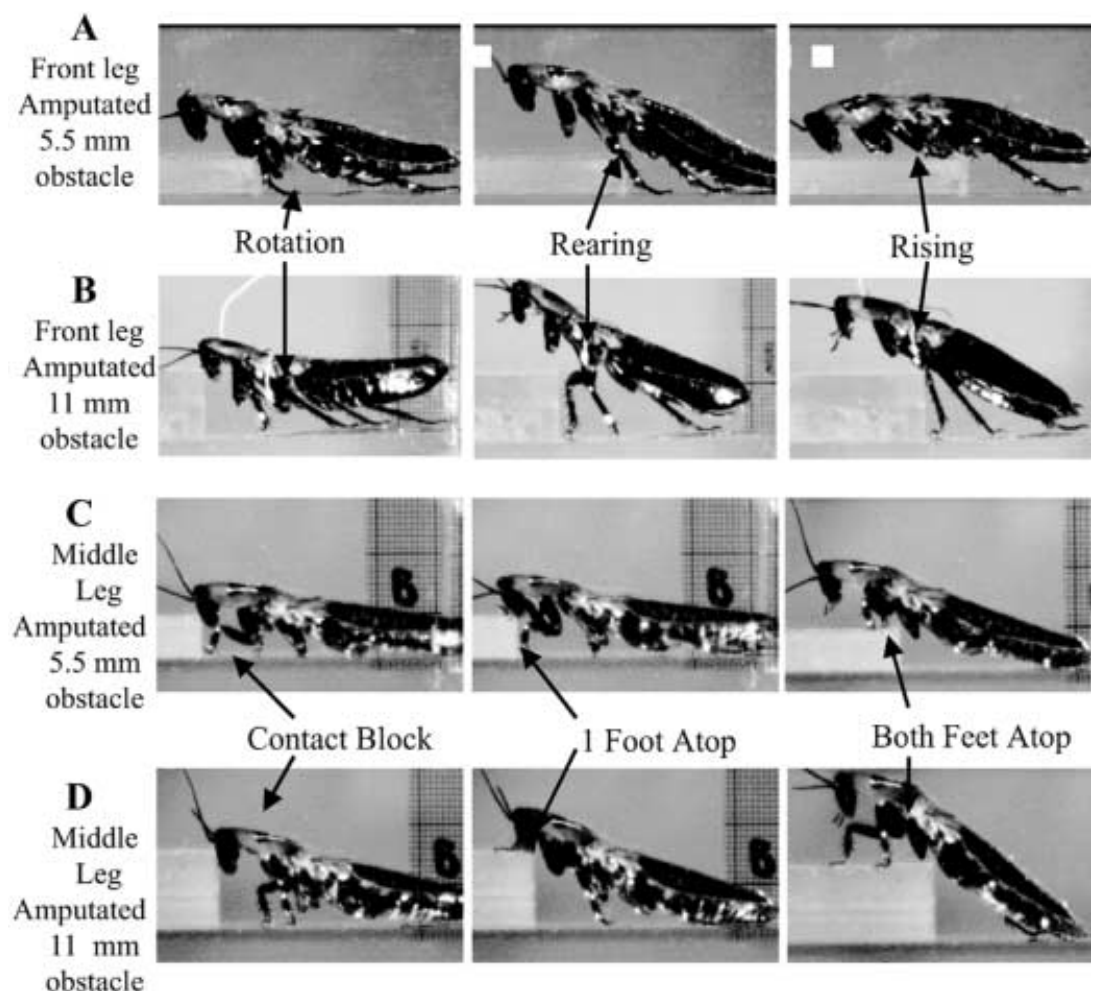

hind leg of three others. This was accomplished by either gluing a minuten pin across the joint on the dorsal surface of the leg or by inserting a minuten pin staple into the trochanter and femur so that it spanned the joint. Neither procedure interfered with normal kinematics of the CTr or FTi joints. High-speed videos of each animal were recorded before and after the trochanter-femur joint was immobilized and the lateral view of the angle between the tibia and the body was compared within animals. In two out of three animals, these procedures reduced tibial rotation of the middle leg during rearing and the hind leg during climbing and leveling $(P<0.05$, Fig. 11$)$ as measured by a decrease in body-tibia angle. In both cases, a decrease was also seen in the third animal, but it was not significant possibly due to high variability in the stapled condition.

\section{Discussion}

Strategies for climbing over blocks of various heights

In order to surmount any obstacle, a legged animal must ultimately displace its CoM upward. For a step-like obstacle, like the blocks used in this study, the problem is compounded by a perpendicular front surface that creates an abrupt transition rather than a gradual incline. Therefore, the animal must drive its CoM to the top of the block with relatively few step cycles. There are a number of strategies that a legged animal could use to accomplish this task. In this study, we found that the deathhead cockroach, B. discoidalis, typically uses two strategies; one for smaller barriers and another for taller ones.

In surmounting barriers that are no more than $5.5 \mathrm{~mm}$ high these cockroaches did not appear to deviate from normal running kinematics. Rather, they took advantage of their normal walking movements in which the swing trajectory of the front leg tarsi reached a height in excess of $6 \mathrm{~mm}$. This means that, confronted with a $5.5-\mathrm{mm}$ barrier, the front leg movements require no changes in order to reach the top of the obstacle. Once one or both front tarsi are placed on top of the block, they can push downward, changing the animal's posture so that the subsequent movements of all legs will drive the CoM upward. Thus, scaling such a barrier is a simple consequence of the encounter with the top of the obstacle rather than an anticipatory change in running strategy. The height to which cockroaches can span barriers in this simple fashion may be extended when they are running at fast speeds where the many-legged sprawled posture provides some dynamic stability. Indeed, Full and his colleagues have reported observations of the same cockroach species running at high speeds over obstacles of varying heights (Full et al. 1998).

At the speeds that we observed, the climbing strategy becomes more complex if the top of the obstacle is beyond the maximum height of the front tarsi during the swing phase of horizontal running. Either the front leg must be lifted higher or the body must be tilted upwards so that at least one front leg can reach the top of the obstacle. Our data indicate that $B$. discoidalis uses the second strategy more often. The critical change for 
A



F Metathoracic spiracle

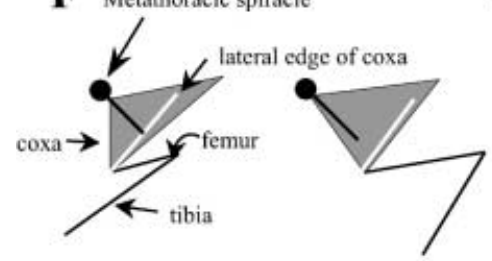

B

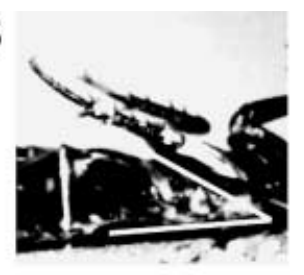

C

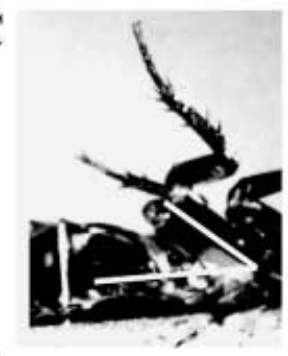

G

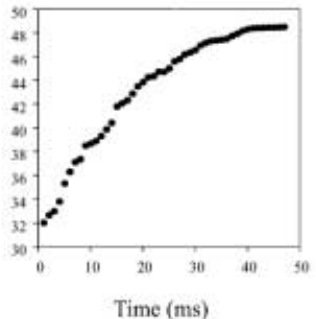

D

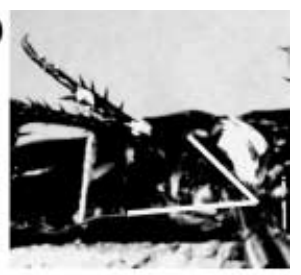

$\mathbf{E}$

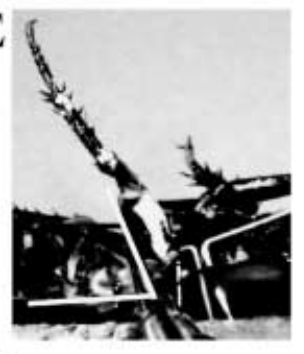

H

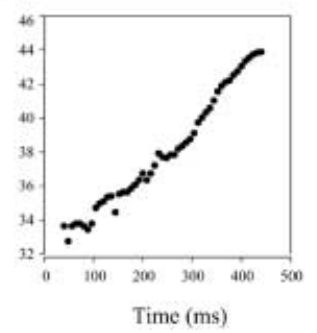

Fig. 9 a The $\alpha$-axis of the thorax-coxa joint (ThC) joint in the middle leg is oriented at a $45^{\circ}$ angle relative to the midline in $B$. discoidalis. Thus, movement of the ThC joint about this axis produces relatively large changes in the orientation of the tibia relative to the body. $\mathbf{b}-\mathbf{e}$ Video images of the $\mathrm{T}_{2}$ leg being passively rotated about the $\alpha$-axis in an anesthetized animal. Images $\mathbf{b}$ and $\mathbf{c}$ were recorded with a camera oriented perpendicular to the animal's body axis to show the lateral view seen in most climbing sequences. Images $\mathbf{d}$ and $\mathbf{e}$ of the same sequence were taken with another camera in line with the $T_{2} \operatorname{leg} \alpha$-axis to show the excursion of this joint. To emphasize the orientation of the coxa, the ventral surface of the coxa was painted white and the lateral edge of the coxa is black. f Stick figures of images from video records indicating markings that were used to quantify the motion of the leg and relate passive movements to active movements. The white line on the coxa indicates the cuticular ridge on the lateral edge of the coxa and the perpendicular black line indicates the distance from the $\mathrm{T}_{3}$ spiracle (large black dot) to the lateral edge. The distance from the first image is superimposed on the second to show the difference that occurs during the movement. g Plot of the change in distance (taken in digital coordinates from the video records) between the cuticular ridge and the $\mathrm{T}_{3}$ spiracle landmarks during passive rotation about the $\alpha$-axis of the ThC joint as in $\mathbf{b}-\mathbf{e}$. $\mathbf{h}$ Plot of the distance between the lateral edge of the coxa and the reference point on the body during the rearing stage of the middle leg. Note the similarity to the passive movement

11-mm obstacles is the rearing stage in which the body tilts upward. In contrast to the climb associated with smaller objects, the animal performs this postural adjustment before the front leg is placed on top of the barrier rather than as a result of stepping onto it. The rearing stage includes extension of the front and middle legs. The middle legs are extended after a rotation to bring them more perpendicular to the ground. When leg orientation is altered in this way, the $\mathrm{CTr}$ and FTi extensions that normally drive the animal forward on a horizontal plane are now associated with changes in the orientation and height of the body. This rearing stage allows the front legs to reach the top of an $11-\mathrm{mm}$ obstacle and starts a cascade of events including rising and leveling stages that bring the animal to the top of the block. A similar rearing stage has been shown to be important for the climbing movements of potato beetles (Pelletier and McLoed 1994) and stick insects (Dürr and Brenninkmeyer 2001).

The altered middle and hind leg kinematics, we have observed, could be accompanied by changes in forces that would not be apparent in kinematic data, as has been noted in turning movements (Jindrich and Full 1999). For example, the cockroach could pull the body upward with the front legs, while the middle legs are pushing downward. Although force measurements were beyond the scope of our study, our kinematic data are largely in agreement with kinetic studies of $B$. discoidalis by Full and co-workers. They also found that to climb a step, the cockroach approached the barrier, tipped the front end of its body up while placing a foreleg onto the step, and then pushed its body over the step. Their data showed greater ground reaction forces produced by middle and hind legs during periods that we refer to as rearing and rising stages (Yamauchi et al. 1993).

Pearson and Franklin (1984) reported some features of the above strategy in locusts walking over ditches or onto blocks. They also noted a characteristic strategy for high obstacles $(1 \mathrm{~cm})$. Locusts paused before the obstacle and changed their posture by flexing the hind 
Fig. 10a-f The trochanterfemur ( $\mathrm{TrF}$ ) joint can produce a change in orientation of the tibia relative to the body. a The trochanter-femur joint is a simple hinge joint. The axis of rotation of the $\operatorname{TrF}$ joint is oriented at approximately $45^{\circ}$ to the long axis of the femur. Rotation about the TrF joint lifts the femur (straight arrow) and moves the leg distal to the trochanter $45^{\circ}$ out of the plane of the CTr and FTi joints (curved arrow). $\mathbf{b}_{1}$ A line drawing of the coxa, trochanter and femur of a $T_{1}$ leg, showing the location of the reductor femoris muscle within the trochanter (shaded region). $\mathbf{b}_{2}$ Longitudinal section through the trochanter and femur showing the bipinnate femoral reductor muscle attached to its central apodeme. $\mathbf{b}_{3}$ Schematic drawing of section in $\mathbf{b}_{2}$, showing the change in orientation of the femur produced by contraction of the reductor femoris muscle. c Direct stimulation of the reductor femoris muscle flexes the $\operatorname{TrF}$ joint. Stimulating electrodes (44-g copper wires, enamel-insulated to the tips) were placed in the reductor femoris muscle in the $T_{1}$ trochanter. The reductor femoris was stimulated and the motion of the femur was video recorded with a camera aimed at the FTi joint oriented parallel to the femur to show the excursion of the $\operatorname{TrF}$ joint. Note the movement in the femur relative to the scale beneath it before (top) and during (bottom) stimulation.

The return is passively actuated by a spring element in the hinge. d Camera lucida drawing of a reductor femoris motor neuron. This neuron was impaled and stimulated intracellularly to produce flexion of the TrF joint and then filled with lucifer yellow
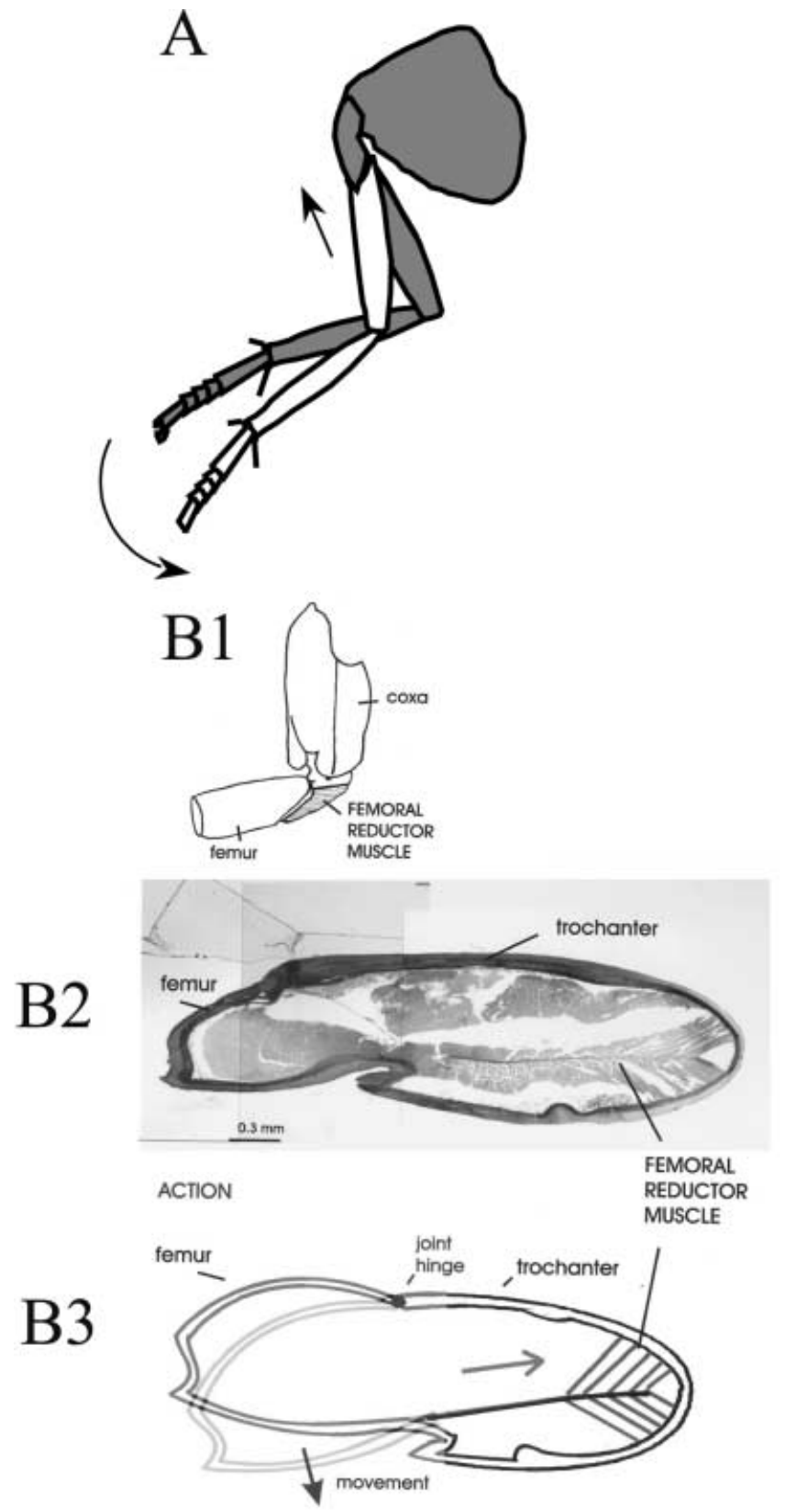

D

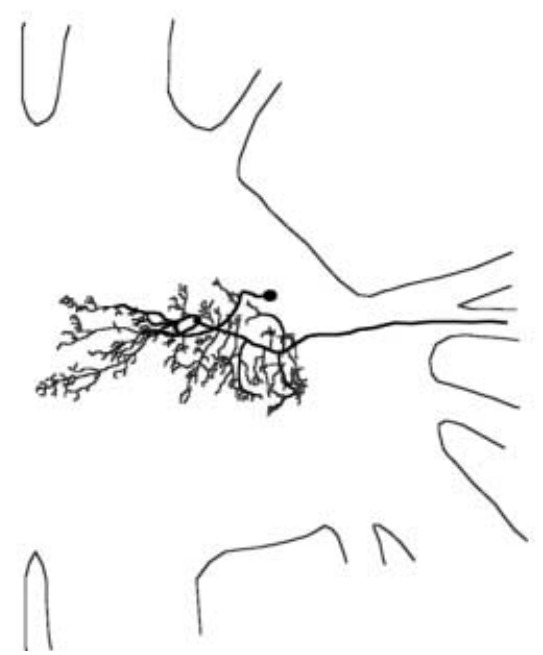

C
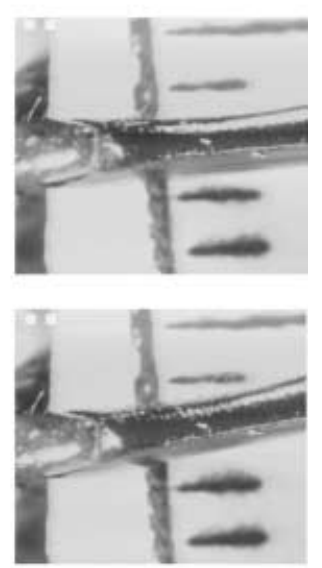
A

Middle Leg (T2)

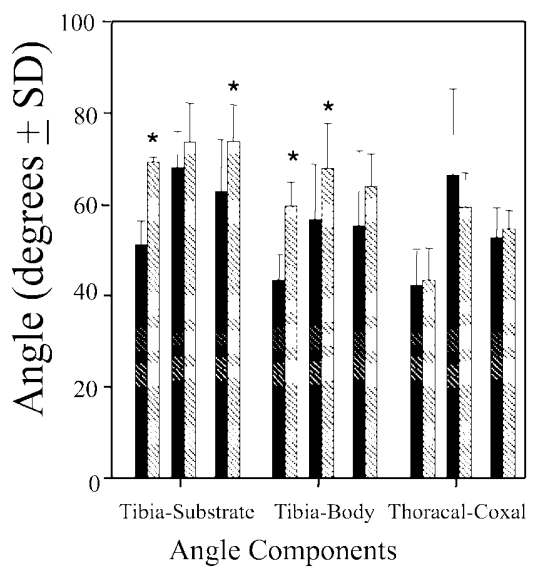

B Middle Leg: TrF Joint Free

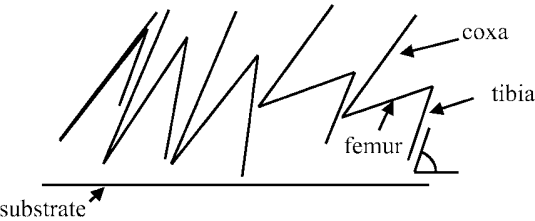

C Middle Leg: TrF Joint Stapled

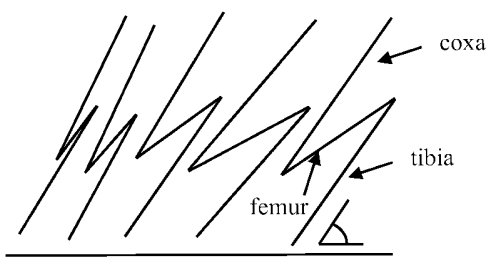

Fig. 11a-f Immobilizing the trochanter-femur joint interferes with the animal directing its tibia vertically. a Two of three animals with staples in their $\mathrm{T}_{2} \operatorname{TrF}$ joint (solid bars) attained a significantly (asterisk: $P<0.05$, stapled versus free) smaller angle between the tibia and the substrate and between the tibia and the body, than animals with the $\operatorname{TrF}$ joint free to move (hatched). Angles were measured at foot set-down of the step in which the animal reared up while approaching the obstacle. ThCs were unaffected in all three animals. In each case separate sets of bars are shown for each of three different animals. Stick figures show the lateral view of the $\mathrm{T}_{2}$ legs unhindered (b) and with the $\mathrm{TrF}$ joint stapled (c). Note that the tibia-substrate angle in the last stick figure, indicated by the angle symbol, is greater in $\mathbf{b}$ than in c. $\mathbf{d}$ Two of three animals with staples in their $\mathrm{T}_{3} \mathrm{TrF}$ joint attained a significantly (asterisk: $P<0.05 ;+: P<0.01$, stapled versus free) smaller angle between the tibia and the substrate and between the tibia and the body. Again no change is seen in the thoracal-coxal joints for any of the animals. Angles were measured at the point when animals placed the $T_{3}$ tibia on the top edge of the block during rising (e). Stick figures show the lateral view of the $T_{3}$ legs unhindered (e) and with the $\operatorname{TrF}$ joint stapled (f). Note the difference in the tibia angle in the last images of the two panels

legs and moving the middle legs to yield a symmetric arrangement that is effectively similar to the rearing stage reported in this study. They also saw simultaneous
D

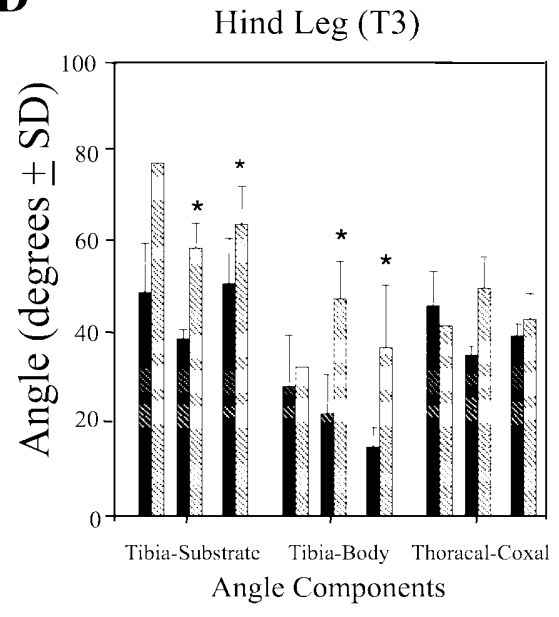

E

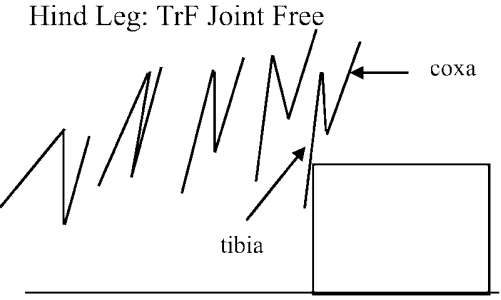

F

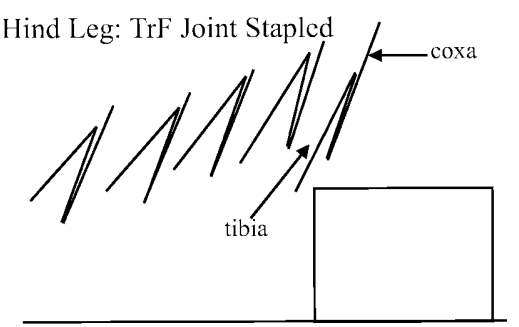

stepping of adjacent ipsilateral legs as the locust ascended onto the block. The cinematographic analysis performed by Pearson and Franklin (1984) used a single top view, so any adjustments in body-substrate angle and some changes in leg orientation would not be evident.

Climbing does not require radical departures from running control mechanisms

The dominant strategy used by $B$. discoidalis to climb required few changes from the leg kinematics used during horizontal running. The vast majority of climbs onto the 11-mm obstacle were accomplished without switching to an entirely new set of leg movements, such as elevating the body by extending all six legs or jumping. The rearing and rising movements used to surmount the $11-\mathrm{mm}$ obstacle involved postural alterations combined with $\mathrm{CTr}$ and FTi extension movements similar to those seen in horizontal running. The departures that we did see from $\mathrm{CTr}$ and FTi running kinematics can all be accounted for by reflex mechanisms controlled within the thoracic ganglia. 
The major alterations in gait during rearing and rising are consistent with the rules for stepping control proposed by Cruse et al. (1995) and Schmitz et al. (2001). They propose that when one leg is experiencing increased loading during stance, the contralateral leg of the same segment delays swing, thereby increasing coactivation of the two legs. In our data, this is seen as an increased tendency for contralateral legs to move in phase with one another during rearing and rising stages of the climb. Although the CTr and FTi joint excursions are unaltered, the duration of stance phase was longer when $B$. discoidalis was climbing. This change may occur because the additional gravitational load experienced during climbing movements delays the time to reach the appropriate joint excursion. The fact that joint excursion during climbing movements is similar to that observed in walking suggests that in both behaviors joint angle detectors shut down the load-induced extensor activity. One well-documented reflex that is active during locomotion in insects is the termination of stance phase by joint angle detectors, such as chordotonal organs (Cruse 1990). An interesting exception is seen in the middle leg FTi joint where excursion is maintained but both the minimum and maximum joint angles are shifted to lower values during climbing.

Reflexes resident in the thoracic ganglia could also control the increased vertical force necessary to lift the body up onto the obstacle. One possibility is that the stance phase motor neurons are reflexively excited and adjusted to the magnitude of forces by campaniform sensilla that detect increased load during the climbing movements. The resultant vigorous activity would augment vertical force development. Similar enhancement of motor activities by sense organs that detect force have been documented in cockroaches and decerebrate cats, some of which may form positive feedback loops. Pearson (1972) recorded from Periplaneta americana walking horizontally while dragging a weight, and found that for a given leg cycle time the greater load produced higher Ds frequency than when the animal was unimpeded. In the decerebrate cats, stimulation of group 1 afferents prolongs stance phase (Whelan et al. 1995), and removal of proprioceptive feedback from leg loading, by the leg stepping into a hole, reduces stance phase extensor activity (Hiebert and Pearson 1999).

\section{Postural changes directed by higher centers}

The aspects of the climbing strategies that appear to require more than thoracic circuitry are the proximal (ThC and TrF) joint movements that initiate the reorientation of the middle legs in preparation for rearing and rising. They are initiated only after the height of the block has been evaluated, presumably by sensors such as eyes and antennae that are located on the head. The choice of postural changes to re-orient first the legs and then the body axis would therefore appear to be directed, at least in part, by the supraesophageal and/or subesophageal ganglia. Furthermore, the swing phase of each leg also may be influenced by head sensors. Unlike the locust and stick insect (Cruse 1980; Pearson and Franklin 1984), cockroaches do not seem to rely on a tarsal elevator reflex to search for the top, but rather assess the obstacle, potentially using visual or antennal inputs, and accurately place the front tarsi upon it.

The changes in orientation of the middle and hind legs seen during climbing occur as a result of relatively small movements at the ThC and TrF joints. Because these joints are located proximally on the leg, small movements can cause a larger rotation at the tibia and tarsus. The role of proximal joints in modifying leg trajectory has been studied in the locust. The body-coxa joint alters hind leg orientation in locusts to convert a jump into a defensive kick (Burrows 1995; Heitler and Burrows 1977a, 1977b). The TrF joint, which had been considered fused, has not been heretofore examined kinematically, although it has been noted that the reductor femoris muscle is present in all insects (Snodgrass 1927). The active function of the $\operatorname{TrF}$ joint during climbing that we describe here, suggests that this joint should be examined more closely.

Perhaps the most remarkable implication of this study is that a discrete set of changes alters locomotion patterns. Movements of the $\mathrm{ThC}$ and $\mathrm{TrF}$ joints need only to be initiated to modify the orientation of the leg. Once this postural change has occurred, normal CTr and FTi joint movements can then take place to tilt the body upward, ultimately placing the front legs within reach of the top of the block. The altered loading on the $\mathrm{CTr}$ and FTi joints can then be compensated for through the fine-tuning of thoracic reflex mechanisms.

Are motor neuron activities in CTr and FTi joints adjusted for climbing?

Although most of the kinematics of the CTr and FTi joints were not significantly altered from those found in running, it does not follow that the motor activity to the muscles that control these joints must also remain the same. Indeed, it is possible that reflexes are invoked to increase or decrease activity in specific motor neurons in order to keep joint excursions within mechanically efficient parameters. We examine this possibility in the companion paper.

Acknowledgements The authors thank Abel Donka, Ryan Edel, Katherine Otto, and Laura Quimby for technical assistance. This research supported by ONR grants N0014-99-I-0378 and N0001496-1-0694. The experiments described in this paper comply with the "Principles of animal care", publication No. 86-23, revised 1985 of the National Institute of Health and also with the current laws of the respective country in which the experiments were performed.

\section{References}

Blickhan R, Full RJ (1993) Similarity in multilegged locomotion: Bouncing like a monopode. J Comp Physiol A 173: 509-517 
Burrows M (1995) Motor patterns during kicking movements in the locust. J Comp Physiol A 176:289-305

Cruse $\mathrm{H}$ (1976) The control of body position in the stick insect (Carausius morosus), when walking over uneven surfaces. Biol Cybern 24:25-33

Cruse H (1980) A quantitative model of walking incorporating central and peripheral influences. I. The control of the individual leg. Biol Cybern 37:131-136

Cruse H (1990) What mechanisms coordinate leg movement in walking arthropods? TINS 13:15-21

Cruse H, Bartling C, Brunn DE, Dean J, Dreifert M, Kinderman T, Schmitz J (1995) Walking: a complex behavior controlled by simple systems. Adapt Behav:385-418

Delcomyn F (1971) The locomotion of the cockroach Periplaneta americana. J Exp Biol 54:443-452

Dresden D, Nijenhuis ED (1953) On the anatomy and mechanism of motion of the mesothoracic leg of Periplaneta americana. Proc Kon Ned Akad Wetensch C 56:39-47

Dürr V, Brenninkmeyer C (2001) The use of the antennae in tactile obstacle localization in the walking stick insect. Congr Neuroethol 5:220

Full RJ, Tu MS (1991) Mechanics of a rapid running insect: two-, four- and six-legged locomotion. J Exp Biol 156:215-231

Full RJ, Blickhan R, Ting LH (1991) Leg design in hexapedal runners. J Exp Biol 158:369-390

Full RJ, Autumn K, Chung JI, Ahn A (1998) Rapid negotiation of rough terrain by the death-head cockroach. Am Zool 38:81 A

Greene SI, Spirito CP (1979) Interlimb coordination during slow walking in the cockroach. II. Effects of cutting thoracic connectives. J Exp Biol 78:245-253

Hiebert GW, Pearson KG (1999) Contribution of sensory feedback to the generation of extensor activity during walking in the decerebrate cat. J Neurophysiol 81:758-70

Heitler WJ, Burrows M (1977a) The locust jump. I. The motor programme. J Exp Biol 66:203-219

Heitler WJ, Burrows M (1977b) The locust jump. II. Neural circuits of the motor programme. J Exp Biol 66:221-241

Hughes GM (1952) The co-ordination of insect movements. I. The walking movements of insects. J Exp Biol 29:267-84
Jindrich DL, Full RJ (1999) Many-legged maneuverability: dynamics of turning in hexapods. J Exp Biol 202:1603-23

Laurent G, Richard D (1986) The organization and role during locomotion of the proximal musculature of the cricket foreleg. I. Anatomy and innervation. J Exp Biol 123:255-283

Marx WJ, Beer RD, Nelson G, Quinn R, Crocker GA (1993) A biomechanical model of the cockroach leg. Soc Neurosci Abstr 19:1601

Nelson GM, Quinn RD, Bachmann RJ, Flannigan WC, Ritzmann RE, Watson JT (1997) Design and simulation of a cockroachlike hexapod robot. Proc ICRA

Pearson KG (1972) Central programming and reflex control of walking in the cockroach. J Exp Biol 56:173-193

Pearson KG (1976) The control of walking. Sci Am 235:72-86

Pearson KG (1993) Common principles of motor control in vertebrates and invertebrates. Annu Rev Neurosci 16:265-297

Pearson KG, Franklin R (1984) Characteristics of leg movements and patterns of coordination in locusts walking on rough terrain. Int J Robot Res 3:101-112

Pelletier Y, McLoed CD (1994) Obstacle perception by insect antennae during terrestrial locomotion. Physiol Entomol 19 360-362

Schmitz J, Dean J, Kindermann T, Schumm M, Cruse H (2001) A biologically inspired controller for hexapod walking: simple solutions by exploiting physical properties. Biol Bull 200:195-200

Snodgrass RE (1927) Morphology and mechanism of the insect thorax. Smithson Misc Coll 80:1-108

Tryba AK, Ritzmann RE (2000) Multi-joint coordination during walking and foothold searching in the Blaberus cockroach. I. Kinematics and electromyograms. J Neurophysiol 83:3323-3336

Watson JT, Ritzmann RE (1998) Leg kinematics and muscle activity during treadmill running in the cockroach, Blaberus discoidalis: I. Slow running. J Comp Physiol A 182:11-22

Whelan PJ, Hiebert GW, Pearson KG (1995) Stimualtion of the group 1 extensor afferents prolongs the stance phase in walking cats. Exp Brain Res 103:20-30

Wilson DM (1966) Insect walking. Annu Rev Entomol 11:103-123

Yamauchi A, Wong B, Kram R, Full RJ (1993) Strategy of scaling steps in insects. Am Zool 33:29A 